\section{Algorithms, Interfaces, and the Circulation of Information: Interrogating the Epistemological Challenges of Facebook}

KOME - An International Journal of Pure Communication Inquiry Volume 4 Issue 1, p. 36-49. (C) The Author(s) 2016 Reprints and Permission: kome@komejournal.com

Published by the Hungarian Communication Studies Association DOI: 10.17646/KOME.2016.13

\author{
Jannick Schou $^{1}$ and Johan Farkas ${ }^{2}$ \\ ${ }^{1}$ IT University of Copenhagen, Denmark \\ ${ }^{2}$ IT University of Copenhagen, Denmark
}

\begin{abstract}
As social and political life increasingly takes place on social network sites, new epistemological questions have emerged. How can information disseminated through new media be understood and disentangled? How can potential hidden agendas or sources be identified? And what mechanisms govern what and how information is presented to the user? By drawing on existing research on the algorithms and interfaces underlying social network sites, this paper provides a discussion of Facebook and the epistemological challenges, potentials, and questions raised by the platform. The paper specifically discusses the ways in which interfaces shape how information can be accessed and processed by different kinds of users as well as the role of algorithms in pre-selecting what appears as representable information. A key argument of the paper is that Facebook, as a complex socio-technical network of human and non-human actors, has profound epistemological implications for how information can be accessed, understood, and circulated. In this sense, the user's potential acquisition of information is shaped and conditioned by the technological structure of the platform. Building on these arguments, the paper suggests that new epistemological challenges deserve more scholarly attention, as they hold wide implications for both researchers and users.
\end{abstract}

Keywords: Epistemology, Facebook, Social Network Sites, Algorithms, Interfaces, Information

\title{
Introduction
}

Through a series of publications, Jessie Daniels has studied so-called cloaked websites (2009a, 2009b, 2014). These are websites in which the authorship and underlying agendas guiding the dissemination of particular contents are deliberately disguised in order to manipulate users. Examples of this type of online propaganda include websites such as 
www.martinlutherking.org (Daniels, 2009a, 2009b) and www.teenbreaks.com (Daniels, 2014). The first of these websites appears to be an educational site about Martin Luther King but is actually run by members of the Ku Klux Klan in order to promote white supremacy. The latter appears to be an educational site about abortion but is actually run by a religious anti-abortion organisation attempting to manipulate users' views on the topic. What both of these sites share, however, is the strategic use of media in order to disguise information under false pretences. They are both ways of sharing and circulating a particular ideological agenda under the guise of either objectivity or political neutrality.

One of the most interesting arguments prompted by Daniels' research has to do with what she terms as emergent "epistemological challenges" linked to the circulation of content through media. Daniels (2014: 143, our emphasis) argues (we quote at length):

The fact that people believe the misstatements, half-truths, and lies on cloaked sites highlights the unique epistemological challenge of activist websites in the digital era. Before the Internet, we relied on a system of gatekeepers such as editors, publishers, broadcasters, and librarians, all of whom mediated information for knowledge seekers. The rise of the popular Internet has not eliminated these gatekeepers, but it has opened a new venue for a kind of publishing that is not mediated by any sort of vetting process. Mostly, this opens new opportunities for a wider range of ideas to be shared by a broader array of groups and individuals; and, at the same time, it raises some disturbing questions about how we acquire and verify knowledge.

According to Daniels' (2014) argument in the quote above, it has becomes increasingly difficult to evaluate how and to what extent information can be regarded as valid or truthful within new mediated environments. New media, Daniels suggests, provides new means to manipulate, filter, and broadcast both truthful and purposely false information. These emergent epistemological challenges are directly tied to reconfigurations in who acts as gatekeepers and how information may be distributed through digital platforms.

In our view, Daniels' (2014) arguments point towards an object of study that is yet to receive a systematic scholarly attention, namely the epistemological challenges introduced by new media at large. Although Daniels (2009a, 2009b) did not frame her analysis in this way, what essentially seems to be at stake in her argument is a question concerning the interplay between technological platforms, understood as complex assemblages of codes, algorithms, design, user behaviour and content, and epistemology. It is, in other words, a question of how information processes - such as the circulation, validation and selection of information - are shaped, modulated, and mediated through the complex socio-technical networks forming inbetween human and non-human actors.

If we take Daniels' arguments seriously, this leads to the introduction of a new and exciting series of questions tied directly to notions of knowledge, information, and media. What is implied by Daniels' reasoning is that fundamental philosophical questions of what we can know and how we can know it - explored from Descartes to Kant and beyond - may take on new and particular forms in the digital era. Yet, at the present moment, it would seem that the full contours of this very wide terrain of inquiry are yet to be properly explicated. How does new media reconfigure and change our ways of evaluating and assessing information? And, equally important, how and to what extend does the adaptation of different media provide us - both as researchers and users - with new epistemological doubts and challenges? 
Questions concerning the ways in which codes, protocols, and algorithms shape and condition our behaviour, potential modes of interaction, and (in the end) subjectivity have increasingly come into view as important topics of research (Berry, 2011; van Dijck, 2013, 2014; Bucher, 2012a, 2012b, 2016a, 2016b; Skeggs \& Yuill, 2015, 2016; Pasquale, 2015; Langlois et al., 2009). Rather than resorting to naïve technological determinism, this body of research - of which the above list is only vaguely indicative - has provided highly productive and often very complex insights into the ways in which new media platforms should not be approached as merely neutral tools, but rather as active agents in their own right. As material environments enveloping our everyday life, media play a highly important part in conditioning our acquisition and evaluation of information. This sentiment is echoed by Milan (2015: 8) in her study of mediated protest: "Evoking the 'material' of (...) social media in the analysis of contemporary collective action has epistemological, ontological, and methodological consequences. It impacts the way in which we can learn about and make sense of contemporary protest".

In this paper, we wish to take the above body of research as our point of departure. Drawing on this research, we will reflect upon and discuss what may broadly be understood as the epistemological dimensions and challenges of information processes on social network sites, specifically in the case of Facebook. We will investigate the conditions for evaluating information, the human and non-human actors selecting, filtering, and circulating information, and the implications of these processes for the evaluation and assessment of information. Rather than a large-scale philosophical argument, we will discuss these questions from a medium-oriented point of view. We wish - to distil our approach to its core - to discuss information construction and circulation from the viewpoint of interfaces, algorithms, and user-platform interactions. By drawing attention to how the underlying code, materiality, algorithms, and interface shape how information is processed, presented, and filtered, we will try to elucidate the connection between epistemology and media technologies. The goal of this paper, then, is to contribute to this emerging field of research by expanding and explicating the range of epistemological questions prompted by new media technologies such as Facebook.

Some key clarifications should be made from the outset of this paper. First of all, we are not using the term epistemology in a strong Kantian sense to imply the internal (cognitive) mechanisms entailed in the processing and understanding of information and senseexperiences. Instead, we attempt to interrogate the ways in which information processes are shaped within complex assemblages of human and non-human actors. Specifically, in the case of this paper, in the context of Facebook as a simultaneously communicative, cultural, and material infrastructure through which information flows are circulated amongst and between human and non-human actors alike. In this sense, our use of the word epistemology does not imply a basically cognitive or psychological frame of reference. Instead, our focus is on the ways in which information can and is processed through both the codes, algorithms, and technological infrastructure provided by Facebook, and the potential engagement and production of information provided by users. This also means that what is attempted here is not a Kittlerian argument concerning the ways in which media has altered and shaped subjectivity across history (Kittler, 1999). In a much more modest way, our intention is to reflect upon the ways in which platforms like Facebook act as active agents in the co-creating and shaping of how information can and is presented, distributed, and made available.

Second, in pursuing this particular perspective, we are not (first and foremost) interested in the various ways in which circulated information may be more or less "true", referring to 
more or less true states. Instead, following an actor-network inspired approach (Latour, 2005), we are much more interested in the dynamic processes entailed in the continuous socio-material configuration and re-negotiation of information. This does not mean that questions of "truthfulness", "representation", and "interpretation" are not valid or important, but simply that they are beyond the initial scope of this paper.

And third, we should also state from the very beginning that a number of the specific claims and arguments made throughout this paper do not necessarily describe "new" features suddenly brought about by the rise of Facebook as an ubiquitous social media. We are, indeed, very sceptical of such claims of radical newness. Yet what is important - and this is the main presumption of this paper - is the notion that Facebook does modulate or reconfigure already-known themes and questions. As Jessie Daniels highlighted in the quotes above, new media can alter the constellation of gatekeepers, algorithms, interfaces and so on, offering new possibilities and conditions for the circulation and evaluation of information. It is precisely this particular modulation that we attempt to explicate in this essay. This, in turn, is the intention of this paper: not to provide a final answer, but to clarify and explicate the ways in which epistemology and the processing of information is modulated with the implementation of new media.

\section{What is Beneath a Page?}

We want to open this paper with an anecdotal example taken from the Danish general elections in 2015 where Danish citizens had to elect their next prime minister as well as the individual Members of Parliament. During this campaign, a screenshot from a post within a secret Facebook group belonging to The Conservative Youth Party was leaked within the Danish mass media. The post, containing a link to a public Facebook page called 'Remember to Vote', read:

CONFIDENTIAL!! We have created this page and Facebook event, where we will try to mobilize young voters during the election campaign by first posting neutral content in the first phase of the election and then posting content related to key issues of The Conservative Party in the last phase ... We really hope that you will support the page and like and share our posts. You are also welcome to invite you friends, also from other parties, so we can reach the wider public, but we underline that it is confidential who is behind the page. (Facebook post, 27 May 2015)

What this post revealed was a sophisticated political tactic: by utilizing a Facebook page in a certain predefined, yet confidential, manner, the Conservative Youth Party would try to manipulate young voters in their direction. They would, as outlined in the secret post, deliberately frame and manipulate the information presented to their audience in a certain pre-conceived way.

This leaked post is interesting for a number of reasons: not just because it showcased how socalled secret groups on Facebook may be utilized in order to organize and carry out hidden operations, but also because it pointed towards a general epistemological problem: how can users know the source and intention behind information distributed and circulated through public Facebook pages? How can we know the underlying procedures governing public information spread through Facebook? 
In this case, it became clear from the leaked information that underneath the public page there was to be a clear-cut strategy for how information should be presented. This information, rather than given in its entirety for the user to evaluate, would be pre-selected and curated in such a way that it would point the users in a specific political or ideological direction. Such withholding of political intention in order to manipulate users is, of course, nothing new, and the question of un-identifiable intentions is not a new one either, as this may also be the case with newspapers, television, books, and other media. However, what is potentially new is the extent to which Facebook - as a material and technological infrastructure - accommodates this form of mal-intentioned distribution of partial information. It is, to put it somewhat crudely, simply very easy for admins to curate, moderate, and selectively present information to users. What is equally important to note is how these technological functions - the ability to curate and create public pages - are no longer tied to particular gatekeepers or uniform centres of power. Instead, they are distributed to a potentially wide range of actors who may use online infrastructures, such as Facebook, to disseminate false or manipulated information.

We will explore the technological dimensions of this argument further below, but for now we wish to simply point towards some broader observations that can be generalized from this anecdotal piece of evidence: namely that what is given, through social media such as Facebook, as public information may be governed by and organized through a whole range of preceding mechanisms. The user cannot be sure that the information is presented in its totality nor if the source of the information is accurate. As we shall return to in the last part of this paper, part of these mechanisms are directly tied to the interface and material constitution of Facebook as a platform. Yet, what these observations also pointed towards was a broader questioning of the conditions and potentials for epistemological doubts related to Facebook and other social network sites. For research, we argue, these observations should be considered carefully. While technological platforms such as Facebook and Twitter offer great opportunities for collecting and analyzing very large corpus of data - in the form of tweets, posts, updates, likes and so on - it remains pertinent that the potential underlying mechanisms governing the production of such information are not forgotten. While indeed difficult to study, we should not be blind to the observation that collective activity may not merely be expressions of spontaneously formed networked publics. There is always the very real option that a number of mechanisms have preceded the publication of information. Underneath the surface, a number of complex epistemological strategies may be at work. If we fail to recognize that Facebook, like all other media (old and new), is not simply an outlet for the dissemination of fully explicated and "neutral" information, but rather the outcome of more or less conscious selection strategies performed by individuals or collectives, then research loses its critical edge. Rather than starting from the presumption that information simply is, we should interrogate how it has come to be what it is.

The case discussed above is an example of intentional withholding and manipulation of information provided by a small group of first and foremost human actors. Yet, as we will argue in this following, these forms of pre-selectivity and concealment of particular information can in fact be seen on a number of different levels in the case of Facebook. In particular, we argue, when looking at the underlying algorithms governing what is represented and representable to the user. It is these issues we turn to in the following section. 


\section{Algorithms and the Selection of Information}

In a recent series of studies, Beverley Skeggs and Simon Yuill $(2015,2016)$ have begun unlocking the underlying set of structural mechanisms governing Facebook's algorithms. To put it somewhat oversimplified, an algorithm can be understood as "a finite list of welldefined instructions for calculating a function, a step-by-step directive for processing or automatic reasoning that orders the machine to produce a certain output from given input" (van Dijck, 2011:30). Through the use of custom-designed software, Skeggs and Yuill have been able to trace how these algorithms work, including how they collect data, manage news, and attempt to redirect attention towards particular forms of content. Skeggs and Yuill (2016: 388) have done so with an emphasis on "areas of activity where a user's own actions came into contact with the actions of the algorithms that Facebook uses to gather information'. Their findings and methodology are both multiple and advanced, and they provide important insights into a series of technological conditions that are often hidden as "business secrets".

A series of findings from Skeggs and Yuill's studies, particularly related to epistemological doubts in the digital age, is how Facebook - in an attempt to maximize their profit and monetization through advertising and collection of data - influence "how your network is shaped over time"' and "how you interact - with whom, when, how" (2016: 391). Skeggs and Yuill (ibid.) show how this is done in what may be termed a reflexive loop in which the platform is "constantly tracking you via Facebook and all other sites (using the APIs)". In short, Facebook's algorithms, named EdgeRank and GraphRank (van Dijck, 2013: 49), curate the content available to the user by filtering out and pre-selecting what and how the user should and ought to see, making "[s]ocial networking sites ... essentially designed and programmable spaces that encourage the user to carry out specific actions" (Bucher, 2012b: 480). This relates to both content from the user's "friends" as well as commercial content via advertising and pages.

In this sense, algorithms do not only constitute the very technological core of Facebook, its machine language, but also "control the "visibility' of friends, news, items, or ideas" (van Dijck, 2013: 49). Thus, "social media platforms do not merely transmit content, but filter it on the basis of claiming to augment it, thereby making the content more relevant to its potential consumers" (O’Callaghan et al., 2015: 460).

At the same time, these algorithms are essentially tied up to the user's actions, communication, and behaviour: the algorithms track, collect, and analyse the user, ensuring a continuous feedback loop between user and platform. As long as the user is present, the algorithm's job is never quite finished. Yet, as Skeggs and Yuill $(2015,2016)$ also show, Facebook's algorithms are not always perfect, and although they will try to match individual behaviour with their pre-defined categories, some individuals may be more difficult to collect and trace than others. Certain forms of subjectivity will be uncategorizable from the perspective of the algorithm. Though multiple algorithms may target different parts of the user's behaviour, the question of algorithms and subjectivity is an important one. If we take Skeggs and Yuill's findings seriously - which suggests that only particular forms of behaviour (i.e. those already expected by the algorithm) will yield the "proper" and "traceable" results - then this also means that embedded within the machine language are pre-existing notions of how subjects should and ought to act. In this sense, the particular algorithm does not only hold an embedded form of normativity - designating a particular outlook on how the subject should act - but also actively re-enforces this pre-conceived notion by operating more or less according to intentions. 
These different considerations connect - in a very real way - to the question of epistemology. The main question prompted by the discussion above is how the individual user can know how presented information has become representable at all. It is, in other words, a question concerning the underlying, algorithmic mechanisms governing what is deemed information (and presented to the user) and what is not. This captures, in many ways, what Gillespie (2014: 168) has called the specific knowledge logic guiding algorithmic assessment of information, which is "built on specific presumptions about what knowledge is and how one should identify its most relevant components."

In this way, rather than simply mediating, the filtering provided by algorithms - based on how the particular user's network has been shaped over time - has implications for what becomes information. Instead of a simple question of information being inputted by one user, only to be transmitted or outputted to all the user's friends, the algorithms are entangled in the modulation and presentation of this information. It is in this precise sense, as mediators rather than intermediaries (Latour, 2005:39), "whose specificity has to be taken into account every time" as their "input is never a good predictor of their output", that algorithms have epistemic implications.

By placing algorithms at the centre of an epistemological questioning, we argue, the focus is shifted from information as knowledge or truth to the underlying processes constituting what appears as information to the user. To put it slightly differently, it is turned from a question of validating already constituted information to a question of how information is pre-selected: what procedures go into constituting what appears as information to the user? And on what particular normative ideals does this pre-selectivity rest? The answer to this question is indeed complex and only partially addressed by the research discussed above. Our aim here is simply to explicate the nature of this question, and, perhaps more importantly, showcase that this is imminently a question concerning always-already entangled relations between human and non-human collectives.

Further expanding this argument, a number of additional considerations should be kept in mind. In the case of Facebook, what is presented as information to the user - after the careful (pre-) selection provided by the algorithms as discussed above - may indeed still be subject to a number of the problems outlined in the first and second section. That is to say, the curative role performed by the algorithms should not necessarily be seen as a filter of more or less trustful information: disinformation or intentionally manipulated information can easily be passed through algorithms. And similarly, in a reverse direction, the pre-selection performed by the algorithm should not be equated with the intentional manipulation performed by individuals, though it does carry similar traits. Specifically in the sense that what is presented to the user as a totality has already been subject to a selection; one whose criteria are not directly negotiable or changeable.

Adding to this last argument, we should keep in mind that the user cannot directly change the algorithms underlying Facebook. There is no switchboard or modular set of controls. If the user wishes to engage actively with the algorithms, she has to engage in careful appropriations and potential purposeful misuses in order to trick, take advantage of, and turn the structural conditions into productive forces, e.g. by 'liking' content that she does not really like, abstaining from certain actions, or by using fake names, images, and so forth. This, however, appears as a completely different scenario: one that is not given in a transparent interplay between user and platform, but rather through a creative exchange that 
hinges on wilful misuse on the user's part. As Milan (2015: 4) argues: 'Users have "mobilized" with some success to counteract this power asymmetry [inherent in Facebook's design], for example, requesting multilanguage services and opposing draconian content moderation policies and design choices; they have occasionally engaged in strategic litigation. But these initiatives do not really have the ability to corrode the regime and the economic model that operate in the back".

So if we accept that Facebook's algorithms play a central part in brokering and engineering what appears as information, then we must also accept, it seems, that this curation of information is never simply the direct output of input provided by other users. Instead, put somewhat simplified, algorithms actively shape the potential information that the user can come to know based on a particular image or normative idea about what counts as information to whom.

\section{The Challenge of Algorithms}

One of the particular problems or challenges regarding algorithms and the technological structure of Facebook is that the technological (and, by extension, algorithmic) dimensions of the platform tend to be or become invisible. As van Dijck (2013: 29) states by referencing Berry (2011: 4): "As software increasingly structures the world, 'it also withdraws, and it becomes harder and harder for us to focus on as it is embedded, hidden, off-shored and merely forgotten about."

We cannot see the software or the algorithms as carriers of particular intentions or ideals. Instead, we tend to simply forget their presence all together. Not only does the algorithms act behind the user's back (so to speak) - which, in the end, is precisely what they are designed to do - but there is also a tendency to forget their existence altogether. This forgetting, withdrawal, or becoming-invisible of technology is not a new argument, as scholars within philosophy, sociology, and information science have discussed the tendency for objects to disappear from our active circumspection for a long time (Heidegger, 1962; Bowker and Star, 1999; Latour, 1996).

However, what is potentially new, in the case of algorithms and platforms like Facebook, is the fact that even if one would want to access and comprehend the algorithms, they would still be largely inaccessible. In the context of Facebook, they remain so-called business secrets, even if research has found ways to reverse engineer or tingle with these systems (Bucher, 2012b; Skeggs and Yuill, 2015). Thus, if a user would want to make them even slightly visible, this would require - as is evident from the complexities of Skeggs and Yuill's studies (2016) - custom-build software or advanced technical proficiency.

In this sense, algorithms are invisible by design, and not just due to inattentiveness on the user's part. As Bucher (2012b: 1176) argues: "One of the problems with analysing algorithms is their often black-boxed nature. While some components of the algorithm are known (...), others remain obscure. We are not seeing completely under the bonnet of the Facebook infrastructure". Thus, Facebook's algorithms and simple tools (like hammers) are not necessarily the same thing. While the hammer may become an object of active circumspection (even if this is not the case for its everyday way of being), algorithms are rather to be regarded as infrastructural elements working within what is accessible to the user 
as a tool: they constitute the very core of the equipment, rather than its immediately apparent features.

While there is certainly a material dimension to this unreadability - a form of invisibility by design - there is also (what we might very loosely term) as a cultural or meaning-bearing component to this withdrawal. One way of framing this is by drawing on what Ernesto Laclau (1990) - following an appropriation of Husserl - names sedimentation. Designating a process of routinization or forgetting, sedimented discourses are arrangements that have taken on the appearance of being natural or ahistorical. Sedimented discourses appear self-sufficient, objective, and are unquestioned. Yet, the point here, according to Laclau (1990), is that such discourses are never merely neutral, but always given through contingent decisions that distinguish between what is included and what is excluded: or, in the case of algorithms, what is represented to the user and what is not, what counts as information and what does not, and how that which is represented (that which is counted) is represented. Sedimentation, then, makes structures appear immobile, unchangeable, and normal, while they are in fact always the product of historical processes with no innate end-purpose or rationale.

This argument can be taken in two directions: first of all, it may be seen as a tendency to forget or neutralize the function of algorithms in our everyday dealings with structures such as Facebook. This argument leans towards a culturalist perspective: what is at stake, in other words, is the perception and articulation of algorithms on the user's part. A second approach, however, may also be to view sedimentation as a material process designating the black boxing of algorithms into neutral and inscrutable voids. In this case, the imperative would be to actively open up and reactivate these sedimented black boxes as material entities in order to grasp the ways in which they shape our potential knowledge about the world. Or as van Dijck (2013: 29, original emphasis) writes: "to make the hidden layer visible and show how software is increasingly quantifying and measuring our social and every day lives".

Summing up these arguments, the key epistemological challenge related to the algorithmic backbone of Facebook is how to make visible that which is invisible by design. This has implications from both a research and a user perspective. Seen from the side of the user, the question is, as we have attempted to outline in the last part of this section, to what extend algorithms take on the appearance of neutrality, to what extent their existence is simply forgotten or rendered neutral. In the case of research, what is at stake is rather how to deneutralize and unpack these algorithmic black boxes - both on a cultural-meaning level and as material entities. Yet, how is this task possible? Is it only computer engineers who can come to grasp the epistemological conditions of the digital age? As we will argue below, a renewed appreciation for particularly the materiality of social network sites may indeed be what is needed.

\section{Opening the Black Box}

When reflecting on the intricacies of social media platforms and their underlying structural conditions, it is easy to get lost in technical language that obfuscates, rather than open up, the area of investigation. In discussing the intimate relations between user behaviour, epistemology, and technological structures, we should not succumb to a turning everything into algorithms, codes, or computational languages, working as silent, complex, and inaccessible machines behind the curtain, tracking, producing, and curating the production of subjectivity. The problem with completely algorithmitizing social media is that it risks 
turning them into black boxes, as discussed above. This argument resonates with a number of existing critical voices, and as Ziewitz states (by reference to Chun, 2008: 300): "Wendy Chun, in particular, has criticized an exclusive focus on computer code as "sourcery," suggesting that 'we need to interrogate, rather than venerate or even accept, the grounding or logic of software", (2016: 7). Indeed, in this regard, we should remember that "[a]lgorithms are but one aspect of Facebook's software assemblage affecting the construction of regimes of visibility and invisibility" as Bucher (2012a: 1177-1778) argues. So while we should algorithmitize our study of social media, this should not lead to a complete reliance on algorithms as the sole mechanisms behind such media. Placing algorithms at the centre of our inquiry should, in other words, not make us reduce social media to algorithms.

A reverse move is to disassemble not only the platforms but also our conceptual vocabularies. Such an approach can be found in the work of van Dijck (2013). Building on actor-network theory (Latour, 2005) as well as political economy (Castells, 1996), she suggests a holistic view on social network sites as given in the interplay between technologies, users, content, business models, governance and ownership. Of particular interest to our discussion of epistemology is her further graduation of the technological dimension into five key components, named (1) (Meta)data, the content and meta-information about the content of the platform; (2) Algorithm, the codes that structure, curate and pre-selects the data and metadata; (3) Protocol, the way the platform is governed through its programing; (4) Interface, what is controllable and observable to the users (i.e. the visible interface) and to the platform owners (i.e. the invisible interface); and (5) Default, automatic predefined settings provided to the user, which steer their actions in certain directions.

What these terms offer is a string of concepts that can be used to dis-entangle the multiple ways in which technological infrastructures, such as Facebook, operate and guide how and what information is processed. The argument that emerges from this conceptual network is the notion that everything cannot be reduced to algorithms, however important they still appear. When discussing questions concerning epistemology, then, we should also be attentive towards these other socio-material components, as they co-structure and coconstruct what and how information can be presented to the user.

In the case of Facebook, a central concern is the extent to which the interface provides different opportunities, conditions, and modes of action depending on the user's role. What we are attempting to get at here is essentially the difference between being a user, a page administrator, and company owners. As we will argue below, each of these roles corresponds to widely different conditions and potential options of information production and circulation. This has implications for our epistemological concerns. In the context of transparency and openness on social network sites, van Dijck (2013: 47) observes the following:

Platform owners have a vested interest in complete openness on the side of the users; the more they know about users, the more information they can share with third parties. (...) However, users' interest is not always served by complete openness; users may want to control third parties' access to the information they voluntarily or involuntarily entrust to Facebook. As a result, Facebook has a stake in promoting the first type of mechanism while diverting attention from the second type: the more users know about what happens to their personal data, the more inclined they are to raise objections. Owners' power over coding technologies thus gives them a distinct advantage over users in the battle for information control. 
While the above quote is dense, its central observation concerns an ambiguity built into Facebook's interface. On the one hand, Facebook wants users to share as much information as possible publicly - public information that can then be collected, aggregated, and passed off to third parties for economic and monetary purposes. On the other hand, however, users do not want to share their information to unknown third parties. So there is a trade-off, on the users' side, between wanting to participate and not wanting to be monetized. The solution for companies such as Facebook, van Dijck (2013) argues, has been to create a distinct hierarchy and knowledge gap between users and platform owners. The interface available to users, in this case, only contains a fraction of the information visible to the company. For example, users cannot know who has access to their content or how it is used.

This knowledge gap is mirrored, albeit in a slightly different way, in the relation between users and page administrators. In being designed for commercial interests, Facebook provides a large set of tools to admins of pages: they can moderate comments, filter out unruly voices, manage data, see information about users, remain completely anonymous, and have a distinct visual hierarchical position on their page (see Schou et al. 2015; Lillqvist et al., 2015). The users, who want to connect and share content, have to accept this fundamentally asymmetrical premise: they can never get to know what the page admins know, as they do not have the same tools available. This technologically-supported dis-symmetry has fundamental consequences for our epistemology interrogation. For if indeed the admin holds the potential to moderate and filter information, then what is presented to the user as e.g. comments on a given page, may, in fact, be only vaguely indicative of the actual totality of content. Furthermore, in being completely anonymously operated, these pages also offer new ways of spreading false information with little to no potential consequences for those behind, other than perhaps their pages being removed by Facebook. Not only is there not a gatekeeper controlling the validity of sources and information, as Daniels (2014) highlighted in relation to cloaked websites, but there is also no way of verifying content and sources. In the end, there is not even potential retaliation towards those spreading such subversive content. So the user operating on Facebook is, indeed, at a distinct disadvantage: a disadvantage that essentially has to do with the material constitution of the platform itself. What the user can and cannot come to know is conditioned by the material structure and potentials inscribed into the platform and its code. Different actors - company owners, page administrators, and users - are positioned within different epistemic positions created through a complex assemblage of code, materiality, commercial interests, and human agency.

Returning to the example presented at the beginning of this paper - the case of the Danish youth party tactically misleading the public - we can see that part of their intentional withholding and manipulation of information was in fact tied to the material possibilities offered by Facebook. Their ability to structure information was tied up with their appropriation of already existing technological potentials. It was because these admits held the ability to curate, moderate, and operate under anonymity that they could manipulate what and how information was presented to potential users. The youth party, in turn, appropriated and exploited the interface provided by Facebook. Now, in this case, algorithms did not provide a prominent feature, yet this does not mean that what was at stake was not the negotiation between human and non-human actors. In this case, it was simply the interface, rather than the algorithms, that provided particular means to control and filter the circulation of information.

Overall, to distil our argument to its very core, what we have tried to unfold in this section is the notion that the pre-selection of information is not only tied to "silent" algorithms. Rather, 
as part of Facebook's interface, there are also numerous potentials for epistemological challenges and uncertainties. Not merely being a neutral tool, the different "subject positions" - admit, user, owner - offered by Facebook co-structures communicative relations and their room for manoeuvrability. This structuring has epistemological consequences for what and how information appears to the user, as different types of users will be able to process information in fundamentally different ways.

\section{Conclusion}

In this paper, we have reflected upon emergent epistemological challenges in the digital era using Facebook as our point of departure. What we have attempted with this discussion has not so much been to provide fully formed answers, but rather to widen the potential range of questions that may be posed in relation to new media technologies. While Daniels (2014) first and foremost pointed towards the epistemological challenges related to deliberate misinformation, we have tried to suggest the always-already present linkage between epistemology, the processing of information, and media. In this sense, our attempt has been to broaden the questioning to a wider set of issues with a wider set of implications. It is important to underline that the arguments made in this paper are obviously not exhaustive and go beyond Facebook as a particular technological structure. Furthermore, we should be conscious of the fact that new media do not simply introduce potential challenges to our perception of the world but also provide new outlets for information exchange, participation, and even protest against oppression. Rather than merely a hindrance, they become a part of already existing and necessarily material epistemological networks conditioning our processing of information. In this sense, new media modulate rather than obfuscate epistemic capacities. With this paper, using Facebook as our particular case, we have attempted to show how this modulation takes places vis-a-vis the underlying algorithmic pre-selection of information, the interface governing interaction on Facebook pages, and the actions performed by human collectives. We have argued that each of these socio-technical processes provide complex epistemological challenges. Yet, we have also attempted to problematize and reflect upon the intricate nature of algorithms in particular, as important objects of research. How can we open up these black boxes without simultaneously fetishizing these as the only matter of concern?

Further reflections on this new field of inquiry, we argue, are of fundamental importance to understanding the implications of information sharing and circulation on social network sites. There is a continuous need to question the complex interrelations between materiality and the processing of information. How do media condition, shape and pre-select the information that we can come to know? How can these socio-technical processes by excavated and disentangled? And how can we develop adequate conceptual vocabularies? As Bucher (2012a: 1778) rightly argues:

What is needed is research that not only attends to changes in editorial media practices as increasingly delegated to algorithms, but also to the changes in cultural assumptions about the nature of social networking that are being built into algorithmic architectures.

With this paper, we hope to have contributed to this exciting field of research by beginning to showcase the complex links between material structures, cultural and communicative practices, and epistemology. 


\section{References}

Berry, D. M. (2010) The philosophy of software: Code and mediation in the digital age. London: Palgrave Macmillan.

Bowker, G. and Star, L. (1999) Sorting Things Out: Classification and Its Consequences. MIT Press.

Bucher, T. (2012a) "Want to be on the top? Algorithmic power and the threat of invisibility on Facebook." New Media \& Society 14(7) 1164-1180. CrossRef

Bucher, T. (2012b) "The Friendship Assemblage: Investigating Programmed Sociality on Facebook." Television \& New Media 14(6) 479-493. CrossRef

Bucher, T. (2016a) "Machines don't have instincts': Articulating the computational in journalism." New Media \& Society First published on January 14, 2016 CrossRef

Bucher, T. (2016b) "The algorithmic imaginary: exploring the ordinary affects of Facebook algorithms." Information, Communication \& Society, First published on February 25, 2016 CrossRef

Castells, M. (2010 [1996]) The Rise of the Network Society: The Information Age: Economy, Society, and Culture Volume, second edition. Oxford: Wiley-Blackwell.

Chun, W. H. K. (2008) 'OOn 'Sourcery,' or Code as Fetish.” Configurations 16 (3) 299-324. CrossRef

Daniels, J. (2009a) "Cloaked websites: propaganda, cyber-racism and epistemology in the digital era." New Media \& Society 11(5) 659-683. CrossRef

Daniels, J. (2009b) Cyber Racism: White Supremacy Online and the New Attack on Civil Rights. New York: Rowman \& Littlefield Publishers Inc.

Daniels J (2014) "From Crisis Pregnancy Centers to Teenbreaks.com: Anti-abortion Activism's Use of Cloaked Websites." In Martha McCaughey (eds.), Cyberactivism on the Participatory Web, pp. 140-154. Routledge.

Gillespie, T. (2014) "The Relevance of Algorthims." In Tarleton Gillespie, Pablo J. Boczkowski and Kirsten A. Foot (eds.), Media Technologies: Essays on Communication, Materiality, and Society, pp. 167-194. London: MIT Press.

Heidegger, M. (1962) Being and Time. Oxford: Blackwell Publishing.

Kittler, F. (1999) Gramophone, Film, Typewriter. Stanford: Stanford University Press.

Laclau, E. (1990) New Reflections on the Revolution of our Time. London: Verso.

Latour, B. (1996) Aramis, or the Love of Technology. Harvard University Press.

Latour, B. (2005) Reassembling the Social: An Introduction to Actor-Network-Theory. Oxford: Oxford University Press.

Langlois, G., Elmer, G., McKelvey, F., and Devereaux, Z. (2009) "Networked publics: The double articulation of code and politics on Facebook." Canadian Journal of Communication 34 415-434.

Lillqvist, E. Louhiala-Salminen, L. Kankaanranta, A (2015) "Power relations in social media discourse: Dialogization and monologization on corporate Facebook pages." Discourse, Context, and Media. First published on November 17, 2015 CrossRef

Milan, S. (2015) "When Algorithms Shape Collective Action: Social Media and the Dynamics of Cloud Protesting." Social Media + Society 1(2) 1-10. CrossRef

O'Callaghan, D., Greene, D., Conway, M., Carthy, J. and Cunningham, P. (2015) "Down the (White) Rabbit Hole: The Extreme Right and Online Recommender Systems." Social Science Computer Review 33(4) 459-478. CrossRef

Pasquale, F. (2015) The Black Box Society: The Secret Algorithms That Control Money and Information. Harvard University Press. 
Schou, J., Farkas, J. and Hjelholt, M. (2015) "The Double Conditioning of Political Participation: Grassroots Politics on Facebook." Conjunctions: Transdiciplinary Journal of Cultural Participation 2(2) 29-47. CrossRef

Skeggs, B. and Yuill, S. (2015) "The methodology of a multi-model project examining how Facebook infrastructures social relations." Information, Communication \& Society, First published on October 20, 2015 CrossRef

Skeggs, B. and Yuill, S. (2016) "Capital experimentation with person/a formation: how Facebook's monetization refigures the relationship between property, personhood and protest." Information, Communication \& Society 19:3 380-396. CrossRef

Van Dijck, J. (2013) The Culture of Connectivity: A Critical History of Social Media. Oxford: Oxford University Press.

Van Dijck, J. (2014) "Datafication, dataism and dataveillance: Big Data between scientific paradigm and ideology." Surveillance \& Society 12(2) 197-208.

Ziewitz, M. (2016) "Governing Algorithms: Myth, Mess, and Methods." Science, Technology, \& Human Values 41(1) 3-16. CrossRef 